Georgian Mathematical Journal

Volume 11 (2004), Number 2, 301-306

\title{
ON ABSOLUTELY NONMEASURABLE ADDITIVE FUNCTIONS
}

\author{
A. KHARAZISHVILI
}

\begin{abstract}
It is shown, under Martin's Axiom, that there exist absolutely nonmeasurable additive functions. Starting with this fact, a generalization of the well-known result of Pelc and Prikry (1983) is obtained. Some related questions concerning the measurability of additive functions are also discussed.
\end{abstract}

2000 Mathematics Subject Classification: 28A05, 28D05.

Key words and phrases: Lebesgue measure, additive function, extension of measure, absolutely nonmeasurable function.

Let $E$ be a set and let $\mu$ be a measure defined on some $\sigma$-algebra of subsets of $E$. We recall that $\mu$ is diffused (or continuous) if all singletons in $E$ are of $\mu$-measure zero. All measures considered below are assumed to be diffused.

Let $f: E \rightarrow \mathbf{R}$ be a function. We say that $f$ is absolutely nonmeasurable if there exists no nonzero diffused $\sigma$-finite measure $\mu$ on $E$ such that $f$ is measurable with respect to $\mu$. The same definition can be introduced for any uncountable Polish space $P$ instead of $\mathbf{R}$.

Example 1. Assume Martin's Axiom (MA). Then it is not difficult to construct a subset $L$ of the real line $\mathbf{R}$, satisfying the following relations:

1) $L$ is a vector space over the field $\mathbf{Q}$ of all rationals;

2) $L$ is a generalized Luzin set, i.e., $L$ is of cardinality continuum $(=\mathbf{c})$ and, for each first category set $X \subset \mathbf{R}$, we have $\operatorname{card}(L \cap X)<\mathbf{c}$.

Taking into account relation 1$)$ and the equality $\operatorname{card}(L)=\operatorname{card}(\mathbf{R})$, we see that $\mathbf{R}$ and $L$ are isomorphic as vector spaces over $\mathbf{Q}$. Let $f: \mathbf{R} \rightarrow L$ be one of isomorphisms between these two spaces. Obviously, $f$ can be regarded as an injective additive function acting from $\mathbf{R}$ into $\mathbf{R}$ (in particular, $f$ is a nontrivial solution of the Cauchy functional equation). Since $\operatorname{ran}(f)=L$ and $L$ is universal measure zero (see, e.g., [1] or [2]), we easily infer that $f$ is an absolutely nonmeasurable function. Thus, there exist solutions of the Cauchy functional equation which are absolutely nonmeasurable. In this context, let us recall the classical fact (within $\mathbf{Z F C}$ theory) that any nontrivial solution of the Cauchy functional equation is necessarily nonmeasurable in the Lebesgue sense.

In the present paper we wish to develop the main idea of Example 1, in order to give its application to the measure extension problem.

Let $\lambda$ denote the standard Lebesgue measure on $\mathbf{R}$ and let $\lambda_{0}$ stand for the restriction of $\lambda$ to the Borel $\sigma$-algebra of $\mathbf{R}$.

The following statement was originally obtained by Pelc and Prikry [3]. 
Theorem 1. Assume the Continuum Hypothesis (CH). Then there exist two $\sigma$-algebras $\mathcal{S}_{1}$ and $\mathcal{S}_{2}$ of subsets of $\mathbf{R}$, such that:

1) the Borel $\sigma$-algebra of $\mathbf{R}$ is contained in $\mathcal{S}_{1} \cap \mathcal{S}_{2}$;

2) both $\mathcal{S}_{1}$ and $\mathcal{S}_{2}$ are countably generated;

3) both $\mathcal{S}_{1}$ and $\mathcal{S}_{2}$ are invariant under the group $\Gamma$ of all isometric transformations of $\mathbf{R}$;

4) there exists a $\Gamma$-invariant measure $\mu_{1}$ on $\mathcal{S}_{1}$ extending $\lambda_{0}$;

$5)$ there exists a $\Gamma$-invariant measure $\mu_{2}$ on $\mathcal{S}_{2}$ extending $\lambda_{0}$;

$6)$ there is no nonzero diffused $\sigma$-finite measure defined on the $\sigma$-algebra generated by $\mathcal{S}_{1} \cup \mathcal{S}_{2}$.

The proof of Theorem 1 given in [3] essentially uses the methods developed in [4] and [5]. Also, in [3] the question is posed whether the assertion of Theorem 1 remains true under Martin's Axiom. By applying the method of Kodaira and Kakutani [6] and starting with Example 1, it will be demonstrated below that the answer to this question is positive (see Theorem 3).

Notice that our approach differs from the one presented in [3] and leads to a much stronger result in terms of absolutely nonmeasurable additive functions.

Let $\mathbf{T}$ be the one-dimensional unit torus in the plane $\mathbf{R}^{2}$. We will consider $\mathbf{T}$ as a compact commutative group, and the group operation in $\mathbf{T}$ will be denoted by + (accordingly, the neutral element in $\mathbf{T}$ will be denoted by 0 ). Note also that $\mathbf{T}$ is equipped with the Haar probability measure $\nu$ which, actually, coincides with the standard Lebesgue measure on $\mathbf{T}$. Further, the group $\mathbf{T}$ is divisible and is representable in the form

$$
\mathbf{T}=G+H \quad(G \cap H=\{0\}),
$$

where $G$ is the countable group of all those elements in $\mathbf{T}$ which have finite order, and $H$ is a complementary subgroup of $\mathbf{T}$.

It can easily be observed that:

a) $H$ is a vector space (over $\mathbf{Q}$ ) isomorphic to $\mathbf{R}$;

b) $H$ is a $\nu$-thick subset of $\mathbf{T}$, i.e., $\nu^{*}(H)=1$;

c) $H$ is a second category subset of $\mathbf{T}$ (moreover, $H$ is thick in the sense of category, i.e., $H$ intersects each second category subset of $\mathbf{T}$ possessing the Baire property).

Taking these facts into account, we have the following auxiliary proposition.

Lemma 1. Under Martin's Axiom, there exists a set $L$ in $\mathbf{T}$ such that:

1) $L \subset H$

2) $L$ is a generalized Luzin subset of $\mathbf{T}$;

3) $L$ is a vector space over $\mathbf{Q}$.

Proof. Indeed, by using the standard argument, a generalized Luzin set $L \subset H$ can be constructed in such a manner that $L$ would also be a vector space over Q (cf. Example 1). We omit the details of this construction (which are not difficult).

The next auxiliary statement plays the key role in our further consideration. 
Lemma 2. Under Martin's Axiom, there exist two functions

$$
\phi: \mathbf{R} \rightarrow H, \quad \psi: \mathbf{R} \rightarrow H,
$$

satisfying the following conditions:

1) $\phi$ and $\psi$ are homomorphisms of vector spaces (over $\mathbf{Q})$;

2) the graph of $\phi$ is $(\lambda \times \nu)$-thick in the product space $\mathbf{R} \times \mathbf{T}$;

3) the graph of $\psi$ is $(\lambda \times \nu)$-thick in the product space $\mathbf{R} \times \mathbf{T}$;

4) $\phi+\psi$ is an isomorphism between $\mathbf{R}$ and $L$, where $L$ is a generalized Luzin set of Lemma 1.

Proof. Let $\alpha$ denote the least ordinal of cardinality continuum and let $\left\{Z_{\xi}\right.$ : $\xi<\alpha\}$ be the family of all Borel subsets of $\mathbf{R} \times \mathbf{T}$ with a strictly positive $(\lambda \times \nu)$-measure. Moreover, fix some partition $\left\{\Xi_{1}, \Xi_{2}, \Xi_{3}\right\}$ of $[0, \alpha[$ into three sets of cardinality continuum and assume that every Borel subset of $\mathbf{R} \times \mathbf{T}$, whose $(\lambda \times \nu)$-measure is strictly positive, belongs to both partial families:

$$
\left\{Z_{\xi}: \xi \in \Xi_{1}\right\}, \quad\left\{Z_{\xi}: \xi \in \Xi_{2}\right\} \text {. }
$$

Let $L$ be as in Lemma 1 . Choose a Hamel basis $\left\{l_{\xi}: \xi<\alpha\right\}$ of $L$. By using the method of transfinite recursion, it is not difficult to construct three $\alpha$-sequences

$$
\left\{x_{\xi}: \xi<\alpha\right\}, \quad\left\{y_{\xi}: \xi<\alpha\right\}, \quad\left\{y_{\xi}^{\prime}: \xi<\alpha\right\},
$$

satisfying the relations:

(a) $\left\{x_{\xi}: \xi<\alpha\right\}$ is a Hamel basis of $\mathbf{R}$;

(b) $\left(x_{\xi}, y_{\xi}\right) \in Z_{\xi}$ for any ordinal $\xi \in \Xi_{1}$;

(c) $\left(x_{\xi}, y_{\xi}^{\prime}\right) \in Z_{\xi}$ for any ordinal $\xi \in \Xi_{2}$;

(d) $\left\{y_{\xi}, y_{\xi}^{\prime}\right\} \subset H$ and $y_{\xi}+y_{\xi}^{\prime}=l_{\xi}$ for each ordinal $\xi<\alpha$.

Now, we define $\phi\left(x_{\xi}\right)=y_{\xi}$ and $\psi\left(x_{\xi}\right)=y_{\xi}^{\prime}$ for all $\xi<\alpha$. In view of the linear independence of $\left\{x_{\xi}: \xi<\alpha\right\}$, the functions $\phi$ and $\psi$ can be uniquely extended to the homomorphisms

$$
\phi: \mathbf{R} \rightarrow H, \quad \psi: \mathbf{R} \rightarrow H,
$$

which also determine the homomorphism

$$
\phi+\psi: \mathbf{R} \rightarrow H .
$$

Since $(\phi+\psi)\left(x_{\xi}\right)=l_{\xi}$ for each $\xi<\alpha$ and $\left\{l_{\xi}: \xi<\alpha\right\}$ is a Hamel basis of $L$, we claim that $\phi+\psi$ is an isomorphism between $\mathbf{R}$ and $L$. Finally, by virtue of relations (b) and (c), it is clear that the graphs of $\phi$ and $\psi$ are $(\lambda \times \nu)$-thick in the product space $\mathbf{R} \times \mathbf{T}$. This ends the proof.

Let $f: \mathbf{R} \rightarrow \mathbf{T}$ be an arbitrary group homomorphism whose graph is $(\lambda \times \nu)$ thick in $\mathbf{R} \times \mathbf{T}$. Then $f$ can be made measurable with respect to an appropriate invariant extension of $\lambda_{0}$ (see, for instance, [6]). Indeed, for each set $Z$ belonging to $\operatorname{dom}\left(\lambda_{0} \times \nu\right)$, denote

$$
Z^{\prime}=\{x \in \mathbf{R}:(x, f(x)) \in Z\}
$$

and consider the family of sets

$$
\mathcal{S}^{\prime}=\left\{Z^{\prime}: Z \in \operatorname{dom}\left(\lambda_{0} \times \nu\right)\right\} .
$$


It is not difficult to verify that $\mathcal{S}^{\prime}$ is a $\sigma$-algebra of subsets of $\mathbf{R}$, containing $\operatorname{dom}\left(\lambda_{0}\right)$ and invariant under the group $\Gamma$ of all isometric transformations of $\mathbf{R}$. Also, we can define a functional $\lambda^{\prime}$ on $\mathcal{S}^{\prime}$ by putting

$$
\lambda^{\prime}\left(Z^{\prime}\right)=\left(\lambda_{0} \times \nu\right)(Z) \quad\left(Z^{\prime} \in \mathcal{S}^{\prime}\right) .
$$

It turns out that $\lambda^{\prime}$ is a measure on $\mathcal{S}^{\prime}$ extending $\lambda_{0}$ and invariant under $\Gamma$ (cf. [6] and [7]). Besides, the definition of $\lambda^{\prime}$ directly implies that the original homomorphism $f$ is measurable with respect to $\lambda^{\prime}$.

Theorem 2. Assuming Martin's Axiom, there exist group homomorphisms

$$
f_{1}: \mathbf{R} \rightarrow \mathbf{T}, \quad f_{2}: \mathbf{R} \rightarrow \mathbf{T}
$$

and two measures $\lambda_{1}$ and $\lambda_{2}$ on $\mathbf{R}$, such that:

1) $\lambda_{1}$ extends $\lambda_{0}$ and is invariant under $\Gamma$;

2) $\lambda_{2}$ extends $\lambda_{0}$ and is invariant under $\Gamma$;

3) $f_{1}$ is measurable with respect to $\lambda_{1}$;

4) $f_{2}$ is measurable with respect to $\lambda_{2}$;

5) the homomorphism $f_{1}+f_{2}$ is absolutely nonmeasurable.

Proof. It suffices to put $f_{1}=\phi$ and $f_{2}=\psi$, where $\phi$ and $\psi$ are as in Lemma 2 . Since the graphs of $\phi$ and $\psi$ are $(\lambda \times \nu)$-thick subsets of $\mathbf{R} \times \mathbf{T}$, they determine the corresponding $\Gamma$-invariant extensions $\lambda_{1}$ and $\lambda_{2}$ of the Borel measure $\lambda_{0}$. At the same time, the group homomorphism $f_{1}+f_{2}$ is injective and its range is a generalized Luzin subset of $\mathbf{T}$. This yields at once that $f_{1}+f_{2}$ is an absolutely nonmeasurable function (cf. Example 1).

Theorem 3. Assume Martin's Axiom. Then there exist $\sigma$-algebras $\mathcal{S}_{1}$ and $\mathcal{S}_{2}$ of subsets of $\mathbf{R}$, satisfying relations 1 )-6) of Theorem 1.

Proof. Let $\mathcal{B}(\mathbf{R} \times \mathbf{T})$ denote the Borel $\sigma$-algebra of $\mathbf{R} \times \mathbf{T}$. Take the homomorphisms $f_{1}$ and $f_{2}$ of Theorem 2 and define:

$\mathcal{S}_{1}=\left\{\left\{x:\left(x, f_{1}(x)\right) \in B\right\}: B \in \mathcal{B}(\mathbf{R} \times \mathbf{T})\right\} ;$

$\mathcal{S}_{2}=\left\{\left\{x:\left(x, f_{2}(x)\right) \in B\right\}: B \in \mathcal{B}(\mathbf{R} \times \mathbf{T})\right\}$.

A simple argument shows that these $\sigma$-algebras are the desired ones. Indeed, relations 1)-5) of Theorem 1 are verified directly. It remains to check the validity of relation 6) of the same theorem. Suppose to the contrary that there exists a nonzero diffused $\sigma$-finite measure $\mu$ on the $\sigma$-algebra generated by $\mathcal{S}_{1} \cup \mathcal{S}_{2}$. Then the homomorphisms $f_{1}$ and $f_{2}$ must be measurable with respect to $\mu$. Consequently, the homomorphism $f_{1}+f_{2}$ must be measurable with respect to $\mu$, too, which contradicts the absolute nonmeasurability of $f_{1}+f_{2}$. The contradiction obtained ends the proof.

Remark 1. One generalization of Theorem 1 for measure type functionals was obtained in [8].

Remark 2. Under Martin's Axiom, Theorems 2 and 3 can be generalized to the case of the $n$-dimensional Euclidean space $\mathbf{R}^{n}(n \geq 1)$ equipped with the group $\Gamma_{n}$ which is generated by the family of all central symmetries of $\mathbf{R}^{n}$. 
Remark 3. In fact, we do not need the full power of Martin's Axiom to obtain Theorems 2 and 3. It suffices to use the corresponding measure-theoretical properties of generalized Luzin sets, which are implied by this axiom.

Remark 4. It is obvious that some additional set-theoretical assumptions are necessary for the existence of absolutely nonmeasurable functions acting from $\mathbf{R}$ into $\mathbf{R}$ (or into $\mathbf{T}$ ). Indeed, if the cardinality of the continuum is real-valued measurable, then there is no absolutely nonmeasurable function acting from $\mathbf{R}$ into $\mathbf{R}$ (into $\mathbf{T}$ ).

Remark 5. Let $E$ be a set and let $f: E \rightarrow \mathbf{R}$ be a function. It can be shown that the following two assertions are equivalent:

a) $f$ is absolutely nonmeasurable;

b) the set $\operatorname{ran}(f)$ is universal measure zero and $\operatorname{card}\left(f^{-1}(t)\right) \leq \omega$ for all $t \in \mathbf{R}$.

In particular, if $\operatorname{card}(E)>\mathbf{c}$, then no real-valued function on $E$ is absolutely nonmeasurable.

The following example is relevant.

Example 2. Let $\mu$ be a $\sigma$-finite measure on $\mathbf{R}$ and let $f: \mathbf{R} \rightarrow \mathbf{R}$ be a function such that, for some nonzero $\sigma$-finite Borel measure $\nu$ on $\operatorname{ran}(f)$, the graph of $f$ is $(\mu \times \nu)$-thick in the product set $\mathbf{R} \times \operatorname{ran}(f)$. Then $f$ is measurable with respect to an appropriate extension $\mu^{\prime}$ of $\mu$ (hence $f$ is not absolutely nonmeasurable). Indeed, we may suppose without loss of generality that $\nu$ is a Borel probability measure on $\operatorname{ran}(f)$. Applying again the method of Kodaira and Kakutani, for each set $Z \in \operatorname{dom}(\mu \times \nu)$ denote

$$
Z^{\prime}=\{x \in \mathbf{R}:(x, f(x)) \in Z\} .
$$

Furthermore, introduce the family of sets

$$
\mathcal{S}^{\prime}=\left\{Z^{\prime}: Z \in \operatorname{dom}(\mu \times \nu)\right\} .
$$

It can easily be seen that $\mathcal{S}^{\prime}$ is a $\sigma$-algebra of subsets of $\mathbf{R}$. In addition, if $X \in \operatorname{dom}(\mu)$, then we have

$$
X \times \operatorname{ran}(f) \in \operatorname{dom}(\mu \times \nu), X=\{x \in \mathbf{R}:(x, f(x)) \in X \times \operatorname{ran}(f)\},
$$

whence it follows that $X \in \mathcal{S}^{\prime}$. Consequently, $\operatorname{dom}(\mu) \subset \mathcal{S}^{\prime}$. Now, for any $Z \in \operatorname{dom}(\mu \times \nu)$ put

$$
\mu^{\prime}\left(Z^{\prime}\right)=(\mu \times \nu)(Z) .
$$

A straightforward verification shows that the functional $\mu^{\prime}$ is well defined (by virtue of the thickness of the graph of our function $f$ ) and that $\mu^{\prime}$ is a measure on $\mathcal{S}^{\prime}$ extending the initial measure $\mu$. The definition of $\mu^{\prime}$ also implies that $f$ is measurable with respect to $\mu^{\prime}$.

In particular, if the graph of a function $f: \mathbf{R} \rightarrow \mathbf{R}$ is $(\lambda \times \lambda)$-thick in the plane $\mathbf{R}^{2}$ (for some examples of such functions, see, e.g., [9] and [10]), then $f$ can be made measurable with respect to an appropriate extension of $\lambda$. In this context, the following question naturally arises. Let $\mu$ be a measure on $\mathbf{R}$ extending $\lambda$ and such that there exists a function from $\mathbf{R}$ into $\mathbf{R}$, whose graph 
is $(\mu \times \lambda)$-thick in the plane $\mathbf{R}^{2}$. Does there exist a group homomorphism from $\mathbf{R}$ into $\mathbf{R}$ whose graph is also $(\mu \times \lambda)$-thick in $\mathbf{R}^{2}$ ? We do not know an answer to this question.

\section{REFERENCES}

1. K. Kuratowski, Topology. I. Academic Press, New York-London; Państwowe Wydawnictwo Naukowe, Warsaw, 1966.

2. A. W. Miller, Special subsets of the real line. Handbook of set-theoretic topology, 201233, North-Holland, Amsterdam, 1984.

3. A. Pelc and K. Prikry, On a problem of Banach. Proc. Amer. Math. Soc. 89(1983), No. 4, 608-610.

4. E. Grzegorek, Remarks on $\sigma$-fields without continuous measures. Colloq. Math. 39(1978), No. 1, 73-75.

5. S. Kakutani and J. Oxtoby, Construction of a non-separable invariant extension of the Lebesgue measure space. Ann. of Math. (2) 52(1950), 580-590.

6. K. Kodaira and S. KaKutani, A non-separable translation invariant extension of the Lebesgue measure space. Ann. of Math. (2) 52(1950), 574-579.

7. A. B. Kharazishvili, Transformation groups and invariant measures. Set-theoretical aspects. World Scientific Publishing Co., Inc., River Edge, NJ, 1998.

8. A. B. Kharazishvili, On countably generated invariant $\sigma$-algebras which do not admit measure type functionals. Real Anal. Exchange 23(1997/98), No. 1, 287-294.

9. B. R. Gelbaum and J. M. H. Olmsted, Counterexamples in analysis. The Mathesis Series, Holden-Day, Inc., San Francisco, Calif.-London-Amsterdam, 1964.

10. A. B. Kharazishvili, Strange functions in real analysis. Marcel Dekker, Inc., New YorkBasel, 2000.

(Received 30.08.2003)

Author's address:

I. Vekua Institute of Applied Mathematics

I. Javakhishvili Tbilisi State University

2, University St., Tbilisi 0143

Georgia

E-mail: kharaz@saba.edu.ge 